\title{
A Quantitative Study of Electrical Stimulation of Central Myelinated Fibers
}

\author{
Spencer L. BeMent and James B. Ranck, Jr. ${ }^{1}$ \\ Bioelectric Sciences Laboratory, Department of Electrical Engineering; and \\ Department of Physiology, The University of Michigan, \\ Ann Arbor, Michigan 78104
}

Received January 29, 1969

Quantitative extracellular electrical stimulation data were obtained from single fibers in the dorsal columns of anesthetized and paralyzed cats. Single-fiber responses were recorded with glass microelectrodes. The threshold current was found for fibers stimulated with a $100-\mu$ diameter monopolar electrode at the surface of the dorsal columns $0.5-2.0 \mathrm{~cm}$ from the recording electrode. Shock artifact was greatly reduced because of the geometry of the stimulating and recording scheme. The distance of the stimulating electrode from a recorded fiber could be determined since fibers in the medial dorsal columns maintain a relatively constant relation to the midline and the surface for several segments. The stimulus was usually a single cathodal current pulse of $50-\mu \mathrm{sec}$ duration. The conduction velocity was also determined for each fiber. Current-distance relations for threshold stimulation were determined for these fibers. In general, the further away the stimulating electrode, the greater the current required for stimulation. Moreover, fibers with larger conduction velocities required less current for stimulation. The fibers were also shown to be periodic in their longitudinal stimulation properties which probably was related to the length of their internodes. The time constant of the nodal membrane was determined from strength-duration data to be about $100-120 \mu \mathrm{sec}$. The mean of the ratio of anodal to cathodal stimulating current was $4.57 \pm 1.00(\mathrm{SD})$ and ranged from 3.19 to 7.70 for $50-\mu \mathrm{sec}$ pulse durations.

\section{Introduction}

Electrical stimulation of the central nervous system is commonly employed experimentally and clinically. However, exactly which elements are stimulated by a given current from a remote electrode is not generally known. One approach to this problem is to find the amount of current necessary to stimulate each kind of neural element (myelinated and unmyelinated fibers, cell bodies, dendrites, etc.) from an electrode a known distance away. From the stimulation properties for all of these types of neural elements, the question of where the actual stimulation occurs in any given

1 This work was supported in part by a research grant from the National Institute of Neurological Diseases and Blindness (NB-04352) and a training grant from the National Institute of General Medical Sciences (NIGMS-5-T01-6M01289-04). 
case can be answered. The effort described here and in the following paper (2) present a fairly complete answer for one type of neural element, the myelinated fiber.

One might think that it would be relatively easy to determine the amount of current necessary to stimulate a particular neural element by merely recording from the element with a microelectrode when the stimulating electrode is located nearby at known distances. However, for small distances between the recording and stimulating electrodes the resulting shock artifact makes such a procedure impractical. Stoney, Thompson, and Asanuma (34) have overcome the shock artifact problem by a clever procedure which uses the abolition of antidromic spikes in pyramidal-tract cell bodies to detect the occurrence of an action potential previously elicited by a stimulus from a nearby microelectrode. Their data deal with electrode distance up to about $150_{\mu}$.

We have overcome the problem of shock artifact by working in the medial part of the dorsal columns where single myelinated fibers maintain a relatively constant relation to the surface and the midline over several segments of length. With this preparation it is possible to record from a single fiber with a microelectrode and to stimulate at a longitudinal distance which is great enough so that shock artifact is not a significant problem. However, the actual distance of the fiber below the stimulating electrode is small and can be determined from a knowledge of the distance of each electrode from the surface and the midline of the dorsal columns. We have obtained current-distance relations for fibers of varions conduction velocities over distances of about $400-1000 \mu$.

In the following paper we develop a model which gives results that agree with the experimental results and which utilizes the data from the dorsal columns as a basis for predicting current-distance relations for other central myelinated fibers. The findings of Stoney $e t$ al. . for units close to the stimulating electrode and our findings for the stimulation of myelinated fibers at larger distances are a start toward a quantitative solution to the problem of electrical stimulation of the central nervous system.

Our two papers also present indirect information about the electrical characteristics of central myelinated fibers. It is often assumed that central myelinated fibers have electrical properties similar to those for peripheral myelinated fibers (19) but there is little data on which to base such an assumption. We give evidence that this assumption is generally correct.

\section{Methods}

Experimental Preparation. The experiments were performed on 45 adult cats under sodium pentobarbital anesthesia. The dorsal surface of the spinal cord was exposed from about $\mathrm{C} 4$ to $\mathrm{T} 4$ and the nonpial layer stripped away 
from two dorsal surface locations that were relatively free of surface blood vessels. One of these sections was later used as a stimulation site, and the pial layer was stripped away from the other section to facilitate the insertion of the microelectrode into the dorsal columns. The cord surface was moistened with warm isotonic saline except during the acquisition of data. 'The animals were stabilized mechanically, and artificially ventilated after paralysis with gallamine triethiodide and bilateral pneumothorax. The core temperature was maintained between 36 and $39 \mathrm{C}$, but no attempt was made to maintain the temperature of the exposed cord except by the warm saline applications. The temperature of the exposed cord surface was $2-5 \mathrm{C}$ less than the core temperature (see the error discussion). The temperature variation in the course of study of any one unit was insignificant.

The stimulating electrodes were platinum-iridium $(90-10 \%)$ wires insulated except for the tip which was ground flat and plated with platinum black. Two $220-\mu$ diameter wires separated by $4.0 \mathrm{~cm}$ were $11 \mathrm{sed}$ for the conduction velocity measurements. The initial studies used an array of four to six $100-\mu$ wires aligned in a row and shaped to conform roughly to the curved dorsal surface, with each electrode approximately $500-\mu$ from its neighbor. However, to overcome the tendency for fluid collection between adjacent electrodes, later experiments used a single $100-\mu$ electrode located at various transverse positions on the dorsal column surface. The indifferent electrode was a rod in the rectum.

The recording electrodes were glass capillary microelectrodes filled with 3 м $\mathrm{KCl}$ to give resistances in isotonic saline solution of $10-30 \mathrm{Mohms.}$ The indifferent electrode was a platinum wire inserted into muscle tissue near the recording site.

The stimulating and recording electrodes were separated by $0.5-2.0 \mathrm{~cm}$ which insured a separation of at least three or four internodal lengths. Therefore, any injury to a fiber at or near the tip of the microelectrode should not have affected appreciably the stimulated node or nodes .

A block diagram of the basic experimental setup is shown in Fig. 1. The stimulus pulses were usually rectangular cathodal current pulses of $50-\mu \mathrm{sec}$ duration with rise and fall times (10-90\%) of less than $5 \mu \mathrm{sec}$. The headstage was a triode cathode follower with neutralized capacitance feedback and had grid current of less than $10^{-10}$ amp. The resistance of the microelectrode was monitored near the end of each oscilloscope sweep. The upper trace of a dual beam oscilloscope was used to monitor the stimulus current intensity. The lower trace consisted of the single-unit response and a delayed pulse which represented the microelectrode resistance.

The actual measurement procedure was approximately as follows. The cord surface was wet with warm isotonic saline for $2-5$ min before each run. The saline was then removed and the surface in the vicinity of the stimulat- 


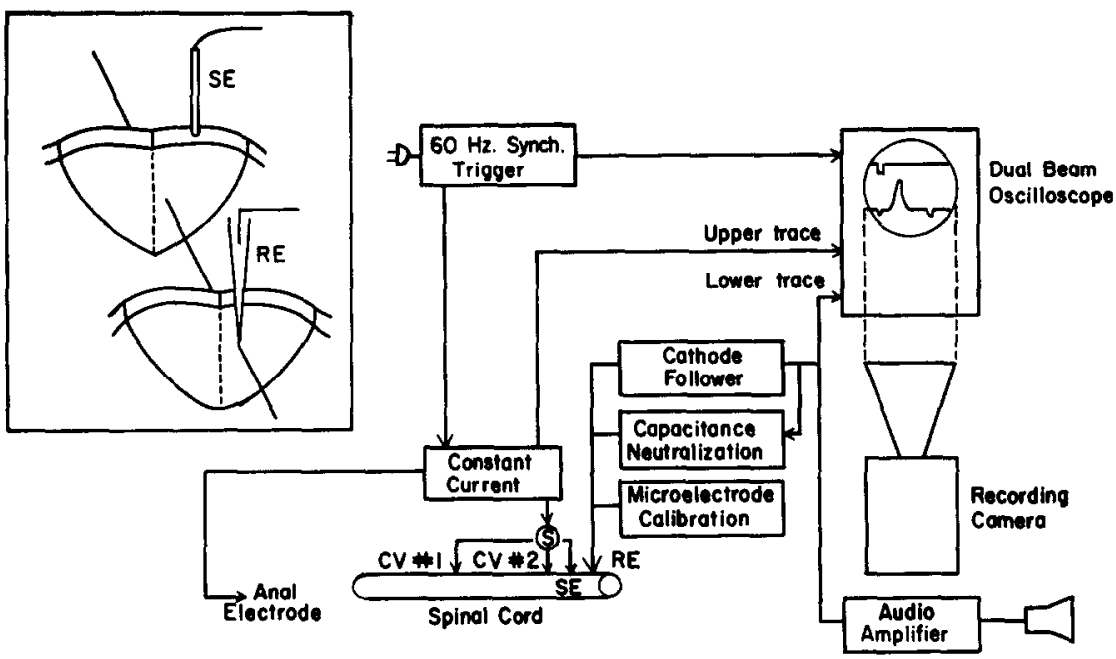

Fir. 1. A block diagram of the hasic experimental setup. CV 1 and CV 2 are the two conduction velocity electrodes. $\mathrm{S}$ is a switch which transfers the stimulating current to the appropriate electrode. SE is the single stimulating electrode or the array electrode used to determine the threshold current-electrode distance relationship. $\mathrm{RE}$ is a glass capillary microelectrode. The other labeled items are described in the text. The inset at the left depicts the dorsal columns with the stimulating and recording electrodes in place. The stimulating electrode was generally $0.5-2.0 \mathrm{~cm}$ caudal to the recording electrode and could be positioned on the columns at various longitudinal and transverse locations.

ing electrode was dried as much as possible. The conduction velocity electrode pair and the stimulating electrode or array were lowered until they rested lightly on the cord surface. The location of the various electrodes relative to the midline was determined with a $20 \times$ dissecting scope and a calibrated eyepiece. Methylene blue in the saline improved visualization of the midline. Stimulating current pulses were delivered from one of the conduction velocity electrodes at $10 / \mathrm{sec}$ and adjusted to trigger many of the fibers in the dorsal columns. The microelectrode was lowered until the tip just came into contact with the surface and the micrometer setting was recorded. The microelectrode was then inserted into the columns until a response of a single unit to the stimulating pulse was noted.

Single units were identified by their sharp responses with distinct thresholds which remained uniform in shape with suprathreshold stimulus strengths. Since the experimental method required only that one recognize when a single fiber was stimulated, it was not necessary to be very selective concerning the size or the site of the response. The responses were generally low amplitude (2-10 mv), although $30-50 \mathrm{mv}$ responses were sometimes recorded. Figure 2 shows the usual positive monophasic character of the re- 


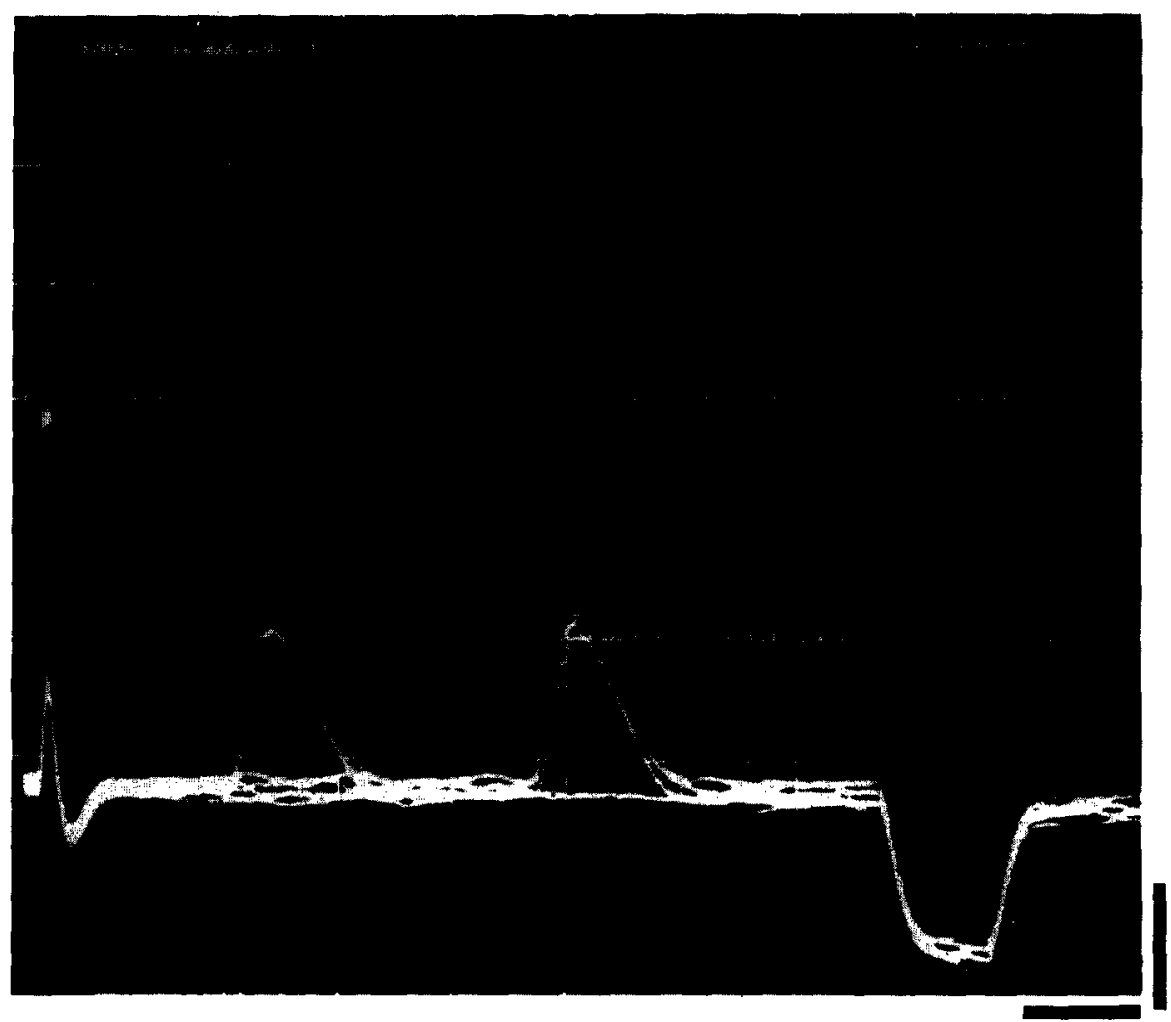

Fig. 2. An example of conduction velocity data. The upper trace shows the $50 \mu \mathrm{sec}$ current pulse at the left and indicates a supramaximal stimulus current of about 155 $\mu$ amp. The lower trace shows the single unit responses resulting from successive stimulation from two electrodes separated by $4.0 \mathrm{~cm}$. The conduction velocity is calculated to be about $30 \mathrm{~m} / \mathrm{sec}$ from the latency difference of $1.33 \mathrm{msec}$. The pulse near the end of the lower trace indicates a microelectrode resistance of about 130 megohms (20 megohms/mv) and an adequate capacitance compensation (see text for details). The small amplitude of the responses shown in the lower trace is probably related in part to the limited high frequency response of the system, as indicated by the relatively long fall time record for the rectangular current pulse used to monitor the microelectrode resistance. A positive voltage at the microelectrode tip corresponds to an upward - leflection of the lower trace. Amplitude calibration is $50 \mu \mathrm{amp}$ for the upper trace and $5 \mathrm{mv}$ for the lower trace. Time calibration is $0.5 \mathrm{msec}$.

sponses. The smaller responses seemed to correspond to those noted by others $(57,60,65)$ who presumed that the tip of their microelectrode had penetrated the myelin laycr but not the axonal membrane, or that the tip dimpled the myelin into the axis cylinder.

Figure 2 illustrates a typical record used to determine the conduction velocity for a single fiber. The five successive traces from each conduction ve- 
locity electrode allow one to average any small variation in latency between the two reponse populations. Fibers which require more than $350 \mu \mathrm{amp}$ of stimulus current from either conduction velocity electrode are not used in order to decrease the chance of stimulating fibers far away from these electrodes.

The stimulus current was then switched to the probe electrode or to one of the electrodes in the array and the stimulus current adjusted to a value just sufficient to trigger the same single fiber for about $50 \%$ of the stimuli. Ten successive stimuli at $1 / \mathrm{sec}$ were photographed, whereupon the stimulating current was switched to the next electrode in the array, or the single probe electrode was moved to a new transverse location. The whole procedure was repeated until threshold values were obtained for the fiber when it was stimulated from several different transverse electrode positions. The usual procedure was to stimulate each time from a new point which was farther from the midline. Data were not taken for electrode locations within $250 \mu$ of the lateral border of the columns to avoid any edge effects. If the microelectrode still remained in the fiber after a complete run, an attempt was made to verify the data by repeating the process. Anodal stimulation data were also obtained from the relatively few preparations which were stable enough so that all of the desired cathodal data had already been obtained. The final step was to note the micrometer reading and withdraw the microelectrode until another micrometer reading for the cord surface could be obtained and averaged with the original surface measurement.

The cord surface was then wet with isotonic saline before another run was attempted. Usually the cord surface was allowed to remain relatively dry for only 15-20 min at a time. Sometimes there was an accumulation of extracellular fluid around the stimulating electrodes which could seriously alter the amount of current required to stimulate a fiber. Therefore, data were selected for analysis only when there was no visible evidence of fluid accumulation. In later experiments a single stimulating electrode was used in place of the electrode array because it was easier to observe any fluid accumulation. However, there did not appear to be any significant difference between the selected array data and the single electrode data.

There was the usual difficulty of prolonged recording from a single fiber. Therefore, whereas conduction velocities were obtained for 322 fibers in 23 cats, stimulation data were obtained from only 101 fibers, and on the average only about three stimulation locations per fiber could be determined.

Sources of Error

Because of the quantitative nature of this study, it is quite important that the known sources of error be presented in detail. Therefore, with the exclusion of the intrinsic variation between animals, the sources of error are 
classified as those related to the anatomical and physiological variability. and those related to the measurement procedure.

i. Errors Related to Anatomical Variations. There are at least four anatomical variations which may be significant. The first is position variability wherein the fiber may change its relative position in a dorsal column over the distance required by the experimental method. We can find no direct experimental data which can be used to describe the possible extent of this variation: however, degeneration studies after sectioning of dorsal roots and parts of the dorsal columns $(14,20,26,27,62)$ seem to give a rather gross indication that the variation is not large over the distances involved. In particular, the figures by Glees, Livingston, and Soler (26) from degeneration studies of single dorsal roots indicate that the variation in position of a single fiber over lengths approximating the distance between our stimulating and recording electrodes may be less than $100 \mu$ in most cases. Moreover, any position variation which does occur is random so that the method of averaging used in the analysis of the data reduces the effect of such variations. Furthermore, the small number of longitudinal stimulation experiments described later indicate variations of less than $100 \mu$ for longitudinal distances of up to $2500 \mu$.

A second anatomical variation is that the fiber diameter is not uniform over the distances involved. This type of variation is reflected in the conduction velocity exhibited by a fiber. Presumably the fiber diameter is not even constant within a given internode, and the different internodes also exhibit some distribution of mean fiber dianeters. Hursh (33) found a mean diameter of $6.5 \pm .47 \mu$ (SD) for a single saphenous nerve fiber traced through $1006-\mu$ serial sections. Duncan (17) noted that single osmicated fibers from sacral and thoracic ventral roots in cat and kitten show diameter variations up to $100 \%$. However, in each case over one-half of the measurements fall within $1 \mu$ of the mean. Distal tapering is known to occur whenever branching occurs in peripheral nerves (18). Other investigators (11, $37,47,49,55)$ detected tapering only with branching in various myelinated peripheral nerves. There is physiological $(42.50)$ evidence that central nbers also exhibit reduced diameters after branching.

A third anatomical variation is that the length between nodes varies along a fiber. Bodian (6) found some irregularity of the nodal spacing in oppossum brain; however, his general impression was one of regylarity approaching that seen in peripheral nerves. In nine axo!s in whirh three or four nodal intervals were measured, only two showe a gross variation of one of the four intervals which approached $50 \%$ of the 'ength of the other three intervals. Fullerton et al. (22) indicated variations in internodal segment lengths in guinea pig lateral popliteal nerves of up to $34 \%$. Hess and Young (32) noted internodal lengths twice or one-hali ff those expected 
from their hypothesized linear relation, which they thought might be the result of obliteration of a node or formation of an extra node. They thought that such irregularities are perhaps more frequent in central fibers. Vizoso and Young (61) also found occasional internodes of twice the normal length in rabbit peroneal fibers. Quilliam and Sato (48) found a wide scatter in internodal lengths of filers from Pacinian corpuscles in cat mesentery, with a tendency for the more proximal internodes to be longer. The limited data on the longitudinal stimulation properties of dorsal column fibers obtained in the current sturly indicate that for some fibers the variation in internodal distance is less than $25 \%$, when followed for three to four internodes.

A fourth anatomical variation is that the location of the stimulated node relative to the stimulating electrode differs for each of the fibers from which the stimulation data were obtained. Our longitudinal stimulation data indicate that the stimulation properties of a fiber are a function of the location of the stimulated node relative to a stimulating electrode. Therefore, two sources of error actually exist here. The first exists because the conduction velocity is a function of the number of nodes or internodal lengths between the two conduction velocity electrodes. Therefore, the effective distance between the sites of stimulation can vary by as much as one internodal length, depending upon the relative location of each conduction velocity electrode with respect to the node which it stimulates. Conduction velocity measurements from fibers for which the distance between the two conduction velocity electrodes was varied in discrete $1.0-\mathrm{cm}$ steps indicate that an electrode separation of $1.0 \mathrm{~cm}$ rest1ts in errors in the computed conduction velocity as large as $75 \%$. When the separation is increased to $4.0 \mathrm{~cm}$, the maximal error decreases to about $10 \%$, which is about what is expected from the anatomical data on internodal lengths. The additional surgery and other complications with the method do not seem to justify a further reduction of this random error by separating the conduction velocity electrodes by more than $4.0 \mathrm{~cm}$. Fortunately, since the location of the nodes with respect to the conduction velocity electrodes should be randomly distributed, averaging of the experimental data within distanct ranges of conduction velocities tends to reduce the size of this error.

The second source of error associated with nodal position variability exists because the measured threshold current for a fiber is a function of the distance of the stimulated node from the stimulating electrode, rather than just a function of the minimal distance of the fiber from the stimulating electrode. Therefore, stimulation data for fiber distances of about one internodal length or less can have large random errors which approach $60 \%$ for distances around $200 \mu$. However, hecause the data are averaged, the effect of these random errors decreases as the sample size increases. 
ii. Errors Related to the Physiological State of the Animal. There are at least three factors which could influence the physiological state of the animal. The first is the effect of the anesthetic on the electrical stimulation and conduction properties of the dorsal column fibers. General anesthetics do not appear to affect conduction in peripheral (40) or central fibers (52, 53 ). We are not aware of any data on the effects of general anesthetics on the excitability of fibers to electrical stimuli.

$A$ second factor which may influence the electrical stimulation and concluction properties of the fibers is the alveolar concentration of carbon dioxide. The fact that the animals were artificially ventilated while anesthetized and paralyzed, and alveolar $\mathrm{PCO}_{2}$, was not monitored means that the alveolar carbon dioxide could have differed from the normal state and, therefore. indirectly have influenced the excitability of the dorsal-column fibers. The effects of carbon dioxide on peripheral and cortical nervous activity have been reviewed (66); however, there appears to be little data concerning the effect of carbon dioxide on the central fibers themselves. Krnjevic, Randic. and Siesjö (38) stated that spinal neurons appear to be less sensitive than cortical neurons to variations in $\mathrm{pCO}^{2}$. They found that $10 \%$ carbon dioxide given for $10 \mathrm{~min}$ failed to alter significantly the submaximal response of a Renshaw cell to ventral-root stimulation, and other synaptic responses recorded inside cells showed no conclusive changes. The resting potential of motoneurons and other spinal neurons was not altered consistently, even by large variations in $\mathrm{pCO}_{2}$. However, Rudin and Eisenman (51), in their in zitro preparation, showed that a total removal of carbon dioxide elicited in central axons a transient enhancement of the resting level of excitability, although this excitability level was depressed with severe or prolonged carbon dioxide removal. They stated that the maintenance of the column spike potential was more critically dependent on carbon dioxide than was the maintenance of the spike potential of its peripheral counterpart.

The uncertainty about the possible influence of alveolar concentration of carbon dioxide upon the excitability of dorsal column fibers to electrical stimuli was somewhat resolved by the following experiment performed on three cats. Each animal was prepared in the same manner as for the usual experiments. Two platinized platinum-iridium (90-10\%) electrodes, $100 \mu$ in diameter and insulated except for the tips, were placed on the surface of a dorsal column close to the midline, and separated by about $0.7 \mathrm{~cm}$ along the midline. The rostral electrode monitored the compound action potential generated by a $50-\mu \mathrm{sec}$ rectangular cathodal current pulse passed through the caudal electrode. The indifferent electrode for both the stimulating and recording electrode was an anal electrode. The size of the compound action 
pound action potential was determined for a given placement of the electrodes, and then the stimulus current was reduced to give a compound action potential of one-third to one-half the maximal amplitude. After a suitable control period, the respiration rate was increased, or the carbon dioxide concentration in the inspired air was increased by attaching a bag of a known amount of carlon dioxide in room air to the intake port of the respirator. The percentage of carbon dioxide in the inspired air was analyzed by a Beckman medical gas analyzer.

The results of the experiments were that increases in the respiration rate of 5 breaths $/ \mathrm{min}$ from basal rates of 19 and 22 breaths/min were followed by increases in the amplitude of the submaximal compound action potential of not more than $10 \%$ within a 5 -min period, after which the amplitude remained relatively constant. Larger increases in the respiration rate from the basal rate generally gave larger increases in the amplitude of the submaximal compound action potential.

The amplitude remained about equal to the control amplitude for carbon dioxide concentrations up to about $2.8 \%$ in the inspired air. Larger concentrations of carbon dioxide usually resulted in a $5-10 \%$ transient increase in amplitude within a few minutes, followed by a rapid decrease in amplitude of $50-60 \% 1$ or 2 min later, at which point the animal was allowed to inspire the normal room air again. ${ }^{2}$ The highest concentration of carbon dioxide inspired was $5 \%$ in room air.

The results of those experiments indicate that the excitability of the dorsal columns to direct electrical stimulation can vary as a function of the alveolar carbon dioxide concentration. However, because of the fact that in the usual experimental situation the respirator was adjusted to hyperventilate the animal slightly, a reduction in excitability corresponding to that seen for carbon dioxide concentrations greater than $2.8 \%$ should not have existed when any of the basic stimulation data were obtained. Furthermore, the respiration rate would have to have been set more than 5 breaths $/ \mathrm{min}$ too high in order to introduce an alteration in excitability between animals of greater than $10 \%$ from this type of variability. The latter situation, while certainly possible, was probably unlikely in most cases.

A third factor which may influence the electrical stimulation and conduction properties of the fibers is temperature. The temperature of the exposed cord surface is generally less than the core temperature by $2-5 \mathrm{C}$. The temperature difference increases toward $5 \mathrm{C}$ as the experiment progresses and also varies between animals. The data of Paintal (45) and Kiraly and

2 The transient increase in excitability and subsequent depression during hypercapnia has been noted for cortical cells (38) and for montoneurons in the spinal cord monosynaptic path $(7,34)$. 
Krnjevic (35) indicate a $Q_{1 \text { of }} 1.6$ for peripheral myelinated nerve fiber conduction velocities in the temperature range of $27-37 \mathrm{C}$. Paintal (44) also indicated that temperature conduction block does not occur at temperatures greater than $25 \mathrm{C}$. If these temperature data are applicable to central fihers, one might expect similar fibers to exhibit about a $10 \%$ variation in conduction velocity because of possible temperature differences. Bishop, Jeremy, and Lance (5) found that when the temperature of the exposed pyramidal tract was decreased from $37 \mathrm{C}$ to as low as $31 \mathrm{C}$, the conduction velocity decreased about $10 \%$ for the $35-40 \mathrm{~m} / \mathrm{sec}$ fiber group. However, Koizumi, Ushiyama, and Brooks (36) indicated that surface temperatures usually were about $5 \mathrm{C}$ lower than the actual temperature inside the spinal cord during cooling. Therefore, it is our belief that the error in conduction velocity contributed by temperature variations within and between animals is probably significantly less than $10 \%$.

Koizumi et al. also noted that cooling the cord did not change significantly the current intensity required to stimulate spinal neurons directly, except in a few cases. However, the rate of current rise required for stimulation was less during hypothermia. Guttman (29) indicated that squid axon under sucrose space clamp had a constant threshold which was relatively independent of temperature for short $(50 \mu \mathrm{sec})$ stimuli. Therefore, we assume that for the stimuli used, the electrical stimulation properties are not altered significantly by variations in the cord surface temperature.

iii. Errors Related to the Measurement Procedure. The sources of error related to the measurement procedure have been estimated to be as follows : (a) The distances of the stimulating and recording electrode from the midline could be determined within about $\pm 50 \mu$, which includes the error associated with the lack of definition of the midline in some animals. (b) The average depth of the microelectrode tip below the surface could be determned within about $\pm 40 \mu$. (c) When these two sources of error are combined, the total error for the actual distance of the fiber from the stimulating electrode is estimated to be less than $\pm 100 \mu .(d)$ The magnitude of the stimulating current could be determined withint $\pm 2 \mu$ amp from the photographs of the scope traces. (e) The error associated with the measurement of the distance between the two conduction velocity electrodes was about $\pm 250 \mu$. ( $f$ ) The error associated with the measurement of the latency difference from the photographic records was about $\pm .02 \mathrm{msec}$.

Another source of error occurs in the determination of conduction velocity since each conduction velocity electrode is not directly above the node which it stimulates. The result of the largest error of this type that can occur is equivalent to an increase or decrease in the electrode separation of one internodal length. Therefore, when the source of error just described is 
combined with measurement errors $(e)$ and $(f)$ above, the result is a total error associated with the determination of conduction velocity that varies from about $\pm 4 \%$ at $20 \mathrm{~m} / \mathrm{sec}$ to about $\pm 8 \%$ at $60 \mathrm{~m} / \mathrm{sec}$.

\section{Results}

Conduction Vclocity Histograms. A histogram of the conduction velocities recorded from 322 fibers in the high thoracic to low cervical regions of 23 cats is shown in Fig. 3. The dotted lines represent the subgroup of conduction velocities of 101 fibers in 20 animals from which the stimulation data were also obtained. The two populations appear to have similar conduction velocity distributions. The median of the large distribution is about $37 \mathrm{~m} / \mathrm{sec}$ and its mean is about $38 \mathrm{~m} / \mathrm{sec}$. The median of the subgroup is also $37 \mathrm{~m} / \mathrm{sec}$ and its mean is about $40 \mathrm{~m} / \mathrm{sec}$.

The paucity of fibers with conduction velocities less than about $22 \mathrm{~m} / \mathrm{sec}$ seems indicative of the fact that it is difficult to record from small-diameter fibers, since anatomical data $(8,9,30)$ indicate that there are many small $(<3 \mu)$ fibers present.

The paucity of fibers with conduction velocities greater than about 57 $\mathrm{m} / \mathrm{sec}$ indicates that the columns do not contain many large-diameter fibers. The application of Hursh's (33) factor of $\operatorname{six}^{3}$ seenns to imply that there

${ }^{3}$ Hursh (33) and Gasser and Grundfest (25) found that the conduction velocity $(\mathrm{M} / \mathrm{sec})$ for cat peripheral myelinated nerve fibers is about six times their fiber diameter $(\mu)$. Other studies on cat optic nerve and tract $(3,4,12,13,41)$ indicate that a factor of six also holds rcasonably well for central myelinated fibers.



Fig. 3. Conduction velocity histograms for dorsal-column fibers. The solid line represents the histogram for 322 fibers in 23 animals for which conduction velocities were determined. The broken line is the histogram for the subgroup of 101 fibers in 20 animals which also provided stimulation data. 
are few fibers present with diameters greater than $9-10 \mu$. In fact, Häggquist (30) found that in man only about $1 \%$ of the fibers of fasciculus gracilis are larger than $10 \mu$.

Average Stimulus Intensity versus Electrode Distance. The relationshins between the average current intensity required to stimulate a fiber and the distance of the stimulating electrode from the fiber are shown in Fig. 4 for four groups of conduction velocities. The actual averaged values, standard errors, and the number of data points from which each average value is determined are indicated in Table 1 . The fact that the data are given at discrete $100-\mu$ distances is explained as follows. For each fiber the stimulating electrode distance is calculated for the various electrode positions, as decribed previously. Then the relationship between threshold current and electrode distance is plotted and a curve fitted to the points by eye. A straight line is drawn between successive points for those cases where there are insufficient data to allow a better approxination. Finally the values at discrete $100-\mu$ distances are abstracted from the plots for each filter and averaged within their conduction velocity group to get the values indicated in Table 1, Fig. 4, and similar figures.

The data directly support the contention that fibers with higher conduction velocities require less current for stimulation, and indirectly support the contention that larger diameter fibers require less current for

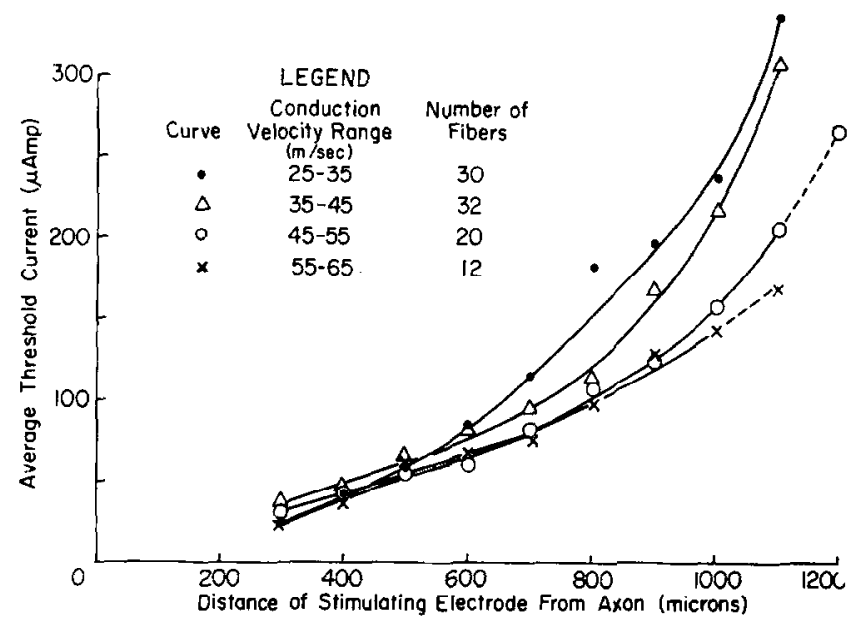

FIG. 4. The relationship between average stimulus current intensity and stimulating electrode distance for various ranges of conduction velocity. Each point represents the average of five or more stimulus intensities, except for the two points connected by dotted lines, which represent three values at $1100 \mu$ and four values at $1200 \mu$. The value for curve (O) at $800 \mu$ would be $160 \mu \mathrm{amp}$ with the exclusion of one distinctly large value of $350 \mu \mathrm{mp}$. The stimulus was cathodal and the pulse duration was 50 $\mu \mathrm{sec}$. The curves were sketched by eye. 


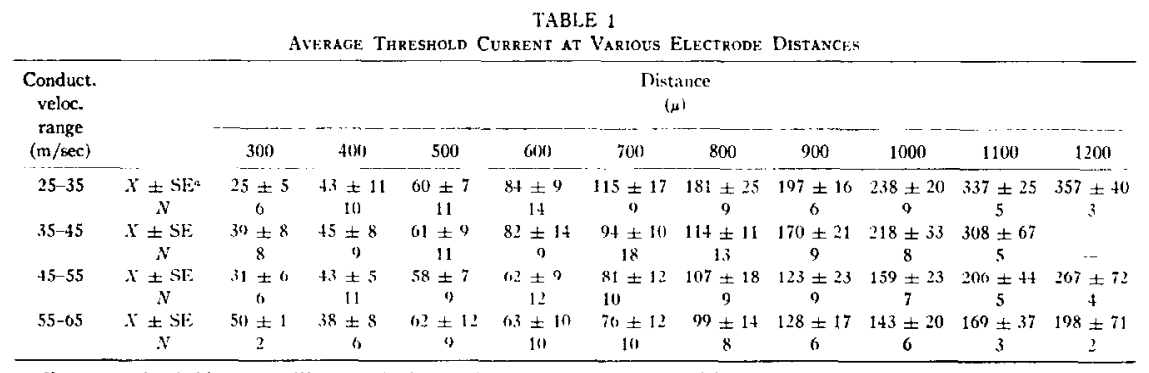

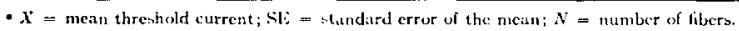

stimulation. ${ }^{4}$ The relationslip between conduction velocity and stimulating current becomes confused for distances less than about $600 \mu$, which can be explained by the increased error present at the smaller electrode distances (see the error discussion in the Methods).

The averaging procedure was necessary because of the large amount of variation that occurred within the raw data for each individual fiber, for the fibers within a single animal, and for the fibers of different animals. In fact. the raw data from each aninal gave only an occasional suggestion that there might be a correlation between conduction velocity and threshold stimulus current. Furthermore, at the smaller electrode distances. something like $15-25 \%$ of the filhers actually required more current for stimulation as the apparent electrode distance decreased. The increased magnitude of the measurement errors at the smaller distances can also explain this apparent discrepancy in some of the data. The threshold stimulus intensity at the larger distances always increased as the distance increased.

The stimulating electrode was never positioned more than about $1 \mathrm{~mm}$ from the midline in the transverse direction to avoid any modification of the current distribution that might be caused by changes in the medium at the lateral edges of the columns. Therefore, in order to obtain data for some of the larger electrode distances, it was necessary to move the stimulating electrode across the midline to the other corsal column. Usually, crossing the midline had no apparent effect upon the threshold stimulus current other than that which would be expected from the increased electrode distance. However, on a few occasions there was a definite discontinuity in the current strength-distance plot that appeared to be associated with the fact that the stimulating electrode was on the other side of the midline. The data for the cases where crossing the midline seemed to have a noticeable effect were not included in the averaged data presented here.

Another Test of the Relationship betreen Conduction Velocity and Stimulus Intensity. The data of Table 1 and Fig. 4 certainly suggest that a signifi-

4 The data shown in Fig. 3 of the following paper (2) for three other conduction velocity groups also support both of these contentions. 
cant relationship exists between the current required for stimulation and the conduction velocity of a fiber. However, in view of the variation in the raw data and the finite possibility that the results may actually reflect some kind of systematic error inherent in the procedure or some systematic change in the state of the animal with time, another test of the proposed relationship was performed.

The same basic procedure for stimulation and recording was employed in this second set of experiments on two animals. The new task was to compare the threshold stimulus intensity with the conduction velocity for two nearby fibers. Therefore, the stimulating electrode was fixed at a single location and data for the conduction velocity and threshold current were secured from a fiber. The microelectrode was then withdrawn from that fiber and inserted into another fiber in the immediate vicinity. Conduction velocity and threshold current data were obtained from this second fiber. Since, in both cases, the stimulating electrode was about the same distance away, it was possible to compare directly the conduction velocity and the threshold current for the two fibers. A particular advantage of this method for relating conduction velocity to threshold current was that there should have been no significant changes in the state of the cord or in the general excitability of the fibers during the $2-5 \mathrm{~min}$ involved in the collection of the data for each fiber pair.

The impalement of the second fiber usually occurred at a new depth within the cord. For the purpose of analysis the data were usually evaluated only for those fiber pairs whose depth did not differ by more than $15 \%$ relative to the smaller fiber depth." The other constraint was that only those fiber pairs with conduction velocities differing by more than $5 \%$ relative to the smaller conduction velocity were analyzed.

The hypothesis proposed is that if two fibers are similarly located with respect to a stimulating electrode, the fiber with the larger conduction velocity requires the smaller threshold current. Therefore, subject to the constraints already discussed, a fiber pair is considered as supporting the hypothesis when the fiber with the larger conduction velocity requires less threshold current than the other fiber of a fiber pair. The first four lines of Table 2 give the results for 21 fiber pairs from two animals.

Each fiber pair can also be considered as composed of one high-conduction velocity fiber and one low-conduction velocity fiber. Then the sign test

\footnotetext{
- However, one situation in which the fiber depths differed by $32 \%$ was included because even though the higher conduction velocity fiber was closer to the stimulating electrode, it still required 38\% more threshold current. Similarly, the data for another fiber pair with a $20 \%$ difference in fiber depth were included because even though the higher conduction velocity fiber was farther from the stimulating electrode, it still reyuired $43 \%$ less threshold current.
} 
can be used to test the null hypothesis that each difference in threshold current between fibers paired on the basis of conduction velocity has a probability distribution with median equal to zero. Line five of Table 2 indicates that this null hypothesis is rejected at the $5 \%$ significance level on the basis of the data from each individual animal and at the $1 \%$ significance level when the data from both animals are pooled.

The data presented in Fig. 4 and Table 2 together can be considered als quite strong evidence for the contention that central fibers with larger conduction velocities require less current for stimulation. Furthermore, the data present strong indirect evidence that central fibers with larger diameters require less current for stimulation.

Longitudinal Stimulation Properties. The longitudinal stimulation properties of dorsal column myelinated fibers were investigated by the determination of the threshold current as the stimulating electrode was moved parallel to the midline in increments of about $125 \mu$. Since about 1 min was required to obtain the data for each stimulating electrode position, it was difficult to record from a single fiber long enough so that the periodicity in the longitudinal stimulating properties could be quantified.

Figure 5 indicates the results for a situation in which the electrical stimulation properties were examined along a longitudinal distance of about 2500 $\mu$, the longest distance obtained in these experiments. A distinct periodicity is indicated which seems to be about $625 \mu$. The apparent overall increase in the minimal threshold current as the stimulating electrode was moved parallel to the midline may be the result of a changing fiber location with respect to the stimulating electrode, an increasing collection of extracellular fluid around the electrode tip, which usually occurs, or both factors acting together.

TABLE 2

Data Associated with the Hypothesis that if Two Fibers are Similarly Located with Respect to a Stimulating Electrode, the Fiber with the Largie Conduction Velocity Requires the Smaller Threshold Stimulation Current

\begin{tabular}{lrrr}
\hline & \multicolumn{3}{c}{ Animals } \\
& 10 & 8 & 18 \\
\hline Pairs which support the hypothesis & 2 & 1 & 3 \\
Pairs which do not support the hypothesis & 12 & 9 & 21 \\
Total pairs & 84 & 89 & 86 \\
Percentage which support the hypothesis & & & \\
Significance level at which the null hypothesis & $\mathbf{5 \%}$ & $\mathbf{5 \%}$ & $1 \%$ \\
$\quad$ is rejected by the sign test
\end{tabular}




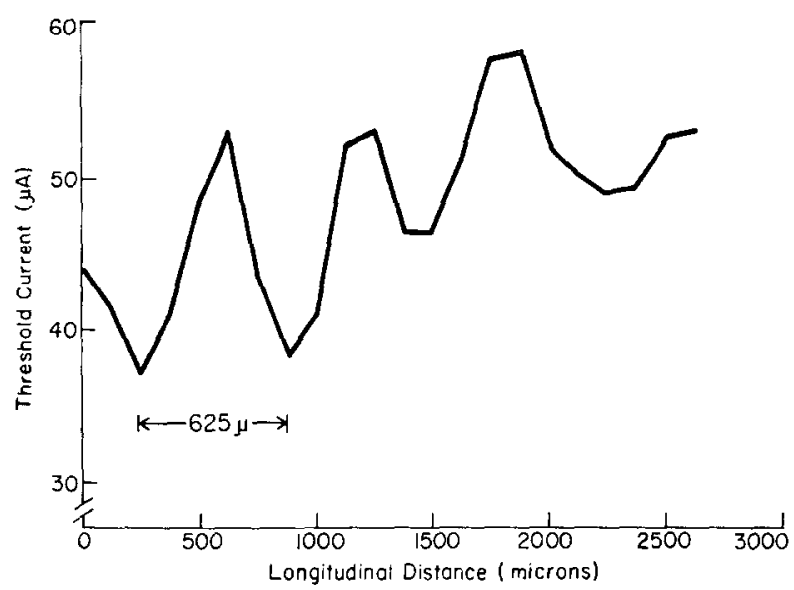

FIG. 5. A plot of the longitudinal stimulation characteristics for a dorsal columm fiber with a conduction velocity of $53 \mathrm{~m} / \mathrm{sec}$. The data were obtained as the stimulating electrode was moved parallel to the midline in a caudal direction away from an initial location arbitrarily defined as zero distance. $50 \mu \mathrm{sec}$ cathodal pulses were used.

The longitudinal stimulation data also indicate that the location of the stimulated node relative to the stimulating electrode has a significant bearing on the amount of current required for threshold stimulation. The fiber of Fig. 5 was located about $300 \mu$ from the stimulating electrode and had peak to valley threshold current ratios of $1.43,1.38$, and 1.25 for the three distinct periods indicated. The mean value of threshold current was about $46 \mu \mathrm{amp}(\mathrm{SE}=1.7 \mu \mathrm{amp})$ for the 25 separate longitudinal locations of the stimulating electrode. The data from six fibers indicate period lengths of $500-1125 \mu$ and peak to valley threshold ratios of $1.25-2.42$. Unfortunately, the small number of fibers investigated indicate no obvious correlation letween conduction velocity and periodicity.

Strength-Duration Curves. Figure 6 presents strength-duration data for four fibers. The chronaxies for these fibers tusually are in the neighborhood of 70-90 $\mu \mathrm{sec}$, which is similar to chronaxies found for peripheral myelinated fibers. No relation between chronaxie and conduction velocity is indicated, although the sample size is certainly limited.

The relation hetween intensity $I_{\mathrm{t}}$ and duration $t$ of threshold stimulating currents is approximately described by the equation

$$
\frac{I_{\mathrm{rh}}}{I_{\mathrm{t}}}=1-\exp \left(-\frac{t}{\tau}\right)
$$

where $I_{\mathrm{rh}}$ is the rheobasic current and $\tau$ is the time constant (21).

Figure 7 presents plots of Eq. [1] for $\tau=75 \mu \mathrm{sec}$ and $\tau=\mu \mathrm{sec}$ in 


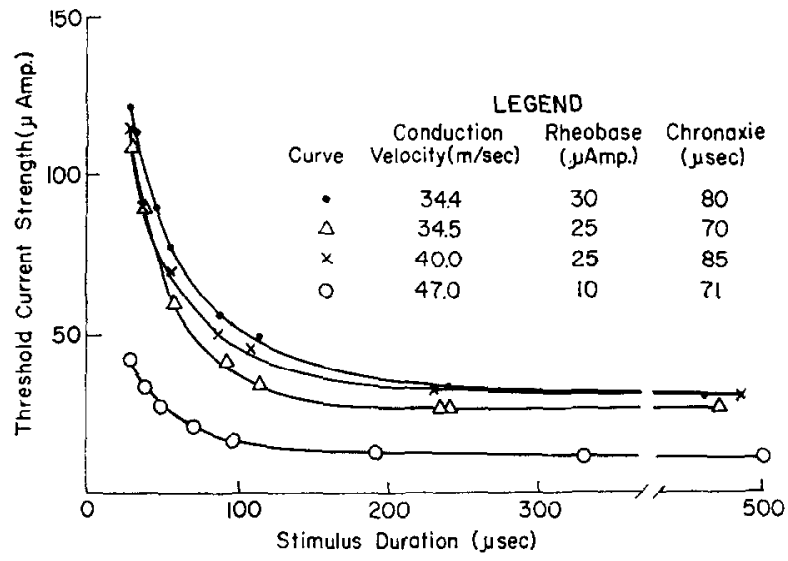

FIG. 6. Strength-duration curves for four dorsal column fibers.

which the curves have been normalized by dividing each $I_{\mathrm{t}}$ by the value of $I_{\mathrm{t}}$ at $t=50 \mu \mathrm{sec}$. The individual points in Fig. 7 are the mean values of the data in Fig. 6, after normalization by dividing the respective threshold currents by $I_{\mathrm{t}}$ at $t=50 \mu \mathrm{sec}$. The normalization procedure allows one to translate the threshold stimulation data obtained for the $50 \mu \mathrm{sec}$ rectangular stimulating pulses into equivalent data for other rectangular pulse durations.

The data of Fig. 7 indicate that the time constant of the nodal membrane may be of the order of $100 \mu \mathrm{sec}$. Furthermore, Eq. [1] can also be solved for $\tau$ and the value of $\tau$ calculated for discrete points from any of the curves of Fig. 6 . Since the three upper curves of Fig. 6 are similar, the mean $I_{\mathrm{t}}$ was found from those curves at $t=25,37.5,50,75,100,150$, and 200 $\mu \mathrm{sec}$ and the value of $\tau$ calculated for those $t$ 's. The mean of the calculated

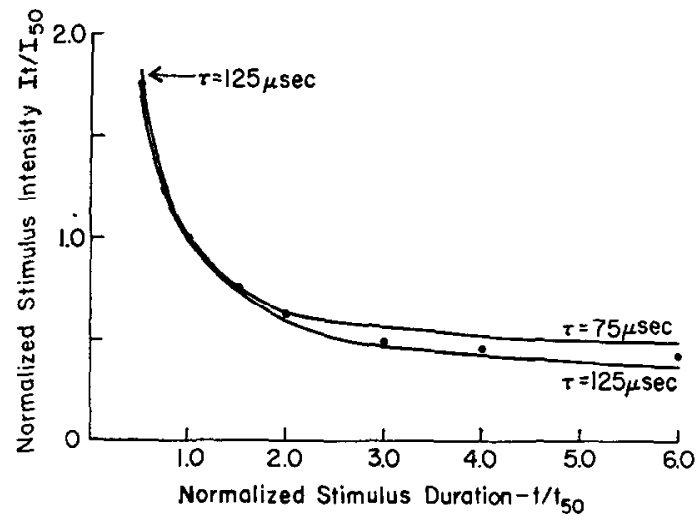

Fig. 7. Normalized strength-duration curves for $\tau=75$ ancl $125 \mu \mathrm{sec}$. The points are the mean values of the data after normalization. 
$\tau$ 's was $107 \pm 4 \mu \mathrm{sec}$ (SD). The mean of the $\tau$ 's at $t=50,100,150$, and $200 \mu \mathrm{sec}$ from the lower curve in Fig. 6 was $112 \pm 17 \mu \mathrm{sec}$ (SD). Therefore, the data of Fig. 7 in combination with the means of the calculated $\tau$ 's indicate that the time constant of the nodal membrane may be about $100-120 \mu \mathrm{sec}$.

Cathodal Versus Anodal Stimulation. The relative efficacy of cathodal and anodal stimulus currents was investigated for nine fibers in six animals. The threshold stimulus was the usual $50 \mu \mathrm{sec}$ rectangular current pulse. The ratio of anodal to cathodal threshold currents was between 3.19 and 7.70 with a mean of 4.57 ( $\mathrm{SD}=1.00)$ from 33 determinations. For any given fiber. each determination was made at a different stimulating electrode location on the dorsal column surface. Stoney ct al. (54) data indicate a ratio of anodal to cathodal threshold current between 3 and 12 (mean $\approx 3.9$ ) for single pyramidal-tract cells in the cat motorsensory cortex.

\section{Discussion}

Conduction Velocities for Dorsal Column Fibers. The maximal conduction velocity for dorsal column fibers is slightly greater than $80 \mathrm{~m} / \mathrm{sec}$ in the macaque (10) and has a mean maximal value of $66.5 \mathrm{~m} / \mathrm{sec}$ with a range of $50-90 \mathrm{~m} / \mathrm{sec}$ in the cat $(28)$.

The mean conduction velocity found in this study was $38-40 \mathrm{~m} / \mathrm{sec}$, in good agreement with mean values for cats noted by other workers $(16,58)$. Some investigators $(15,59,64)$ found mean values closer to $50 \mathrm{~m} / \mathrm{sec}$. The range of conduction velocities found in this study agrees well with the range of $25-65 \mathrm{~m} / \mathrm{sec}$ found by Uddenberg (59). Several workers $(23,24$, $42,50,63$ ) noted that many of the large fibers in the dorsal columns seem to end or branch in the upper lumbar to lower thoracic region, and some fibers apparently exhibit a further decrease in conduction velocity in the upper thoracic to lower cervical region $(23,50,63)$. Therefore, the conduction velocities recorded in this study at these higher levels seem to be generally consistent with the findings of others.

The histogram of Fig. 5 appears to be the most extensive physiological data available for the conduction velocity spectrum of dorsal column fibers. However, the selection bias discussed earlier should be taken into account in any interpretation of these data.

Other Longitudinal Stimulation Data. Ono ${ }^{6}$ demonstrated that the threshold for a single excised nerve fiber showed a sharp minimum at every node of Ranvier, when measured with a $10-100-\mu$ diameter stimulating electrode moved along the fiber. Lussier and Rushton (43) stimulated a frog sciatic nerve (stripped of epineurium) by a moveable cathode, with a grounded

${ }^{6}$ The information concerning the work of Ono was obtained from Tasaki (57) and is presumably from peripheral nerve fibers. 
anode very close on each side, and found that excitation occurred only at certain points on the fiber, apparently when the cathode was over a node. They concluded that medullated nerve fibers in situ are only excitable at the nodes. Tasaki (56) stated that the current intensity necessary to excite a single motor fiber near the surface of the intact frog ventral root shows a sharp minimum at every node of Ranvier.

Tasaki (57) moved a $25 \mu$ (ID) glass capillary stimulating electrode along the pial surface of the dorsal funiculus of a partially isolated frog spinal cord. His results indicated a variation of threshold with distance, with the points of minimal threshold spaced fairly regular at $200-300 \mu$, and an occasional spacing of $400 \mu$ or more. Furthermore, at points halfway between the points of minimal threshold, the fiber was practically insensitive to the stimulating current. His method did not provide information concerning the distance of the fiber from the stimulating electrode or the conduction velocity for the fiber. Otherwise, the results of his studies are consistent with the findings associated with Fig. 5.

Cathodal and Anodal Stimulation. The mean (4.57) of the ratio of anodal to cathodal threshold currents found in this study is similar to the mean (3.9) calculated from the data of Stoney et al. (54) for single pyramidal tract cells in the cat motor-sensory cortex. However, they used a $200-\mu \mathrm{sec}$ current pulse.

The difference between the amount of current required to stimulate a fiber from a monopolar anode and from a monopolar cathode is usually explained as follows. The actual stimulation occurs when an outward directed current depolarizes a nodal membrane enough to bring it into the active state, thereby generating an action potential. ${ }^{7}$ Furthermore, if the stimulating cathode is located somewhere between two adjacent nodes, it seems reasonable that more cathodal current would be required to trigger an action potential at one of the nodes than if the cathode is located directly above a node.

For the case of a monopolar anode, the current direction is reversed. Therefore, the outward-directed stimulating current is distributed throughout many nodes so that less current crosses any one node in an outward direction for the same stimulating current intensity as in the cathodal case. Consequently, more total anodal than cathodal stimulating current is required to generate an action potential.

Some of the relationships between the location of a stimulating electrode relative to a node, the polarity of that electrode, and the excitability of a fiber have been derived and experimentally verified by Lussier and Rushton (43) for frog sciatic nerve fibers. However, their work assumes that the

\footnotetext{
7 Figure 1 of the next paper presents a schematic diagram of the current flow linies
} associated with a cathodal stimulating electrode located directly above a node. 
stimulating electrode is immediately adjacent to the fiber; therefore, their results cannot be readily applied to the data presented here.

The Strength-Duration Relationship. We have not found any other data which describe the strength-duration relationship for central myelinated fibers, although such data may exist. The data shown in Fig. 6 are certainly small in quantity; however, there seems to be little reason to believe that additional data would alter the normalized data appreciably. Furthermore, Woodbury (64) oltained strength-duration curves for ten sciatic nerve fibers with conduction velocitics of $20-10 \mathrm{~m} / \mathrm{sec}$. His individual curves were indistinguishable from each other or from the curve for the entire bundle, and the average time constant of excitation was $180 \mu \mathrm{sec}$. Frank and Fuortes (21) found by direct stimulation that cat ventral root fibers have an average time constant of $270 \mu \mathrm{sec}$ and a chronaxie of $190 \mu \mathrm{sec}$.

Other Quantitative Central Stimulation Studics. There have been a few studies which present data that indicate general relationships between the threshold stimulating current and the distance of the stimulating electrode from the stimulated element. Landgren. Phillips, and Porter (39) examined the threshold to cortical stimulation of pyramidal cells by recording from single pyramidal axons at the $\mathrm{C} 5-\mathrm{C} 6$ level in baloons. The form of their curves seems to be in general agreement with the curves of Fig. 4 , although a direct comparison is not possible because they stimulated cortical structures from a surface anode with $200-\mu$ sec pulses. Phillips ( 46 ) and Hern, Phillips, and Porter (31) performed similar studies for cortical Betz cells in the cat. Microstimulation within the depth of the cortex of cats has been used to

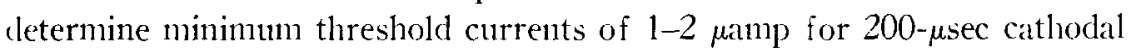
current pulses $(1,54)$. Stoney ot al. (51) found that the threshold current seemed to be parabolic function of the distance of the stimulating electrode from the cell for distances up to $150 \mu$. Their work is discussed in greater detail in the next paper.

\section{References}

1. Asanuma, H. and H. Sakata. 1967. Functional organization of a cortical efferent system examined with focal depth stimulation in cats. $J$. Neurophysiol. 30 : 35-54.

2. BeMent, S. L. and J. B. RANCK, JR. 1969. A model for electrical stimulation of myelinated central fibers with monopolar electrodes. Exptl. Neurol. 24: 171186.

3. Bishop, G. H. and M. H. Clare. 1955. Organization and distribution of fibers in the optic tract of the cat. J. Comp. Neurol. 103: 269-304.

4. Bishop, P. O., D. Jeremy, and J. W. Lance. 1953a. The optic nerve. Properties of a central tract. J. Phy'siol. London 121 : 415-432.

5. Bishop, P. O., D. Jerexry, and J. W. Lance. 1953b. Properties of pyramidal tract. J. Neurophysiol. $16: 537-550$. 
6. Bodian, D. 1951. A note on nodes of Ranvier in the central nervous system. J. Comp. Neurol. 94: 475-483.

7. Brooks, C. McC. and J. C. Eccl.es. 1947. A study of the effects of anaesthesia and asphyxia on the monosynaptic pathway through the spinal cord. J. Neurophysiol. $10: 349-360$.

8. Bunge, R. P., M. B. Bunge, and H. Ris. 1960. Electron microscopic study of demyelination in an experimentally induced lesion in adult cat spinal cord. $J$. Biophys. Biochem. Cytol. 7: 685-696.

9. BunGE, R. P. and P. M. GLAss. 1965. Some observations on myelin-glial relationships and on the etiology of the cerebrospinal fluid exchange lesion. Ann. N.Y. Acad. Sci. 122: 15-28.

10. Carrea, R. M. and H. Grundfest. 1954. Electrophysiological studies of cerebellar inflow. I. Origin, conduction and termination of ventral spinocerebellar tract in monkey and cat. J. Neturophysiol. 17: 208-238.

11. Causey, G. 1948. The effect of pressure on nerve fibres. J. Anat. $82 ; 262-270$.

12. Chang, H-T. 1952. Functional organization of central visual pathways. Res. Publ. Assuc. Res. Nervous Mental Disease 30: 430-453.

13. Chang, H-T. 1956. Fiber groups in primary optic pathway of cat. J. Neurophysiol. 19 : 224-231.

14. ChAng, H-T. and T. C. Ruch. 1947. Organization of the dorsal columns of the spinal cord and their nuclei in the spider monkey. J. Anat. 81: 140-149.

15. Collins, W. F. and C. T. Randt. 1956. On electrophysiological study of small myelinated axons in anterolateral column in cat. J. Neurophysiol. $19:$ 438-445.

16. DiBiagio, F., and H. Grundfest. 1955. Afferent relations of inferior olivary nucleus. II. Site of relay from hindlimb afferents into dorsal spino-olivary tract in cat. J. Neurophysiol. 18 : 299-304.

17. Duncan, D. 1934. A relation between axone diameter and myelination determined by measurement of myelinated spinal root fibers. J. Comp. Neurol. $60: 437-471$.

18. Eccles, J. C. and C. S. Sherrington. 1930. Numbers and contraction values of individual motor units examined in some muscles of the limb. Proc. Roy. Soc. London Ser. B. 106 : 326-357.

19. Eisenman, G. and D. O. Runin. 1954a. Further studies on the functional properties of spinal axons in sivo. J. Gen. Physiol. 137: 495-503.

20. Ferraro, A. and S. E. Barrera. 1935. Posterior column fibers and their termination in macacus rhesus. J. Comp. Neurol. $62: 507-530$.

21. Frank, K. and M. F. G. Fuortes. 1956. Stimulation of single motoneurones with intracellular electrodes. J. Physiol. London 134: 451-470.

22. Fullerton, P. M., R. W. Gilliatt, R. G. Lascelles, and J. A. MorganHughes. 1965. The relation between fiber diameter and internodal length in chronic neuropathy. J. Physiol. London 178: 26-28P.

23. Gardner, E., F. Latimer, and D. Stillweli. 1949. Central connections for afferent fibers from the knee joint of the cat. Am. J. Physiol. 168: 437-441.

24. Gasser, H. S., and H. T. Graham. 1933. Potentials produced in the spinal cord by stimulation of dorsal roots. Am. J. Physiol. 103:303-320.

25. Gasser, H. S., and H. Grundfest. 1939. Axon diameters in relation to the spike dimensions and the conduction velocity in mammalian A fihers. An. J. Phy.riol. 127: 393-414.

26. Glees, P., R. B. Livingston, and J. Soler. 1951. Der intraspinale Verlauf und die Endigungen der sensorischen Wurzeln in den Nucleus Gracilis und Cuneatus. Arch. Psychiat. Nervenkr. 187 : 190-204. 
27. Glefs, P., and J. Soler. 1951. Fibre content of the posterior columns and synaptic connections of nucleus gracilis. $Z$. Zellforsch. $36: 381-400$.

28. Grundfest, H., and B. Campbell. 1942. Origin, conduction and termination of impulses in dorsal spinocerebellar tracts of cats. J. Neurophysiol. 5 : 275-294.

29. Gutrman, R. 1966. Temperature characteristics of excitation in space-clamped squid axons. J. Gen. Physiol. $49: 1007-1018$.

30. HäGGQvist, G. 1936. Analyse der Fäserverteilung in einem Rückenmarkquerschnitt (th3). Z. Mikrosk. Anat. Forsch. $39: 1-34$.

31. Hern, J. E. C., C. G. Phillips, and R. Porter. 1962b. Electrical thresholds of unimpaled corticospinal cells in cat. Quart. J. Exptl. Physiol. 47: 134-140.

32. Hess, O. and J. Z. Young. 1949. Correlation of internodal length and fibre diameter in the central nervous system. Nature 164: 490-491.

33. Hursir, J. B. 1939. Conduction velocity and diameter of nerve fibers. Am. J. physiol. 127: 131-139.

34. King, C. E., W. E. Garrey, and W. R. Bryan. 1932. The effect of carbon dioxide, hyperventilation, and anoxemia on the knee jerk. Am. J. Physiol. 102: 305-318.

35. Kiraly, J. K. and K. KRnjevic. 1959. Some retrograde changes in function of nerves after peripheral section. Quart J. Exptl. Physiol. 44: 244-257.

36. Koizumi, K., J. Ushiyama and C. McC. Brooks. 1960. Effect of hypothermia on excitability of spinal neurons. J. Neurophysiol. 23 : 421-431.

37. KREINDLER, A. 1927. Relation entre le diametre des fibres nerveuses et al chronaxie chez la grenouille. Compt. Rend. Soc. Biol. 97: 125-127.

38. Krnjevic, K., M. Randic, and B. K. Siesjö. 1965. Cortical $\mathrm{CO}_{2}$ tension and neuronal excitability. I. Physiol. Iondon 176: 105-122.

39. Landgren, S., C. G. Phildips, and R. Porter. 1962. Cortical fields or origin of the monosynaptic pyramidal pathways to some alpha motoneurons of the baboon's hand and forearm. J. Physiol. London 161: 112-125.

40. Larrabee, M. G., and J. M. Posternak. 1952. Selective action of anesthetics on synapses and axons in mammalian sympathetic ganglia. $J$. Ncurophysiol. 15: 91-114.

41. Lennox, M. A. 1958. Single fiber responses to electrical stimulation in cat's optic tract. J. Neurophysiol. $21: 62-69$.

42. Lloyd, D. P. C., and A. K. MCIntyke. 1950. Dorsal column conduction of group I muscle afferent impulses and their relay through Clarke's column. J. Ncurophysiol. 13 : 39-54.

43. Lussiek, J. J. and W. A. H. Rushron. 1952. The excitability of a single fibre in a nerve trunk. J. Physiol. London 117: 87-108.

44. Paintal, A. S. 1965a. Block of conduction in mammalian myelinated nerve fibres by low temperatures. J. Physiol. London 180: 1-19.

45. Paintal, A. S. 1965b. Effects of temperature on conduction in single vagal and saphenous myelinated nerve fibres of the cat. J. Physiol. London 180: 20-49.

46. Phillips, C. G. 1956. Cortical motor thresholds and the threshold and distribution of excited Betz cells in the cat. Quart. J. Exptl. Physiol. 41 : 70-84.

47. Quilimam, T. A. 1956. Some characteristics of myelinated fibre populations. $J$. Anat. 90: 172-187.

48. Quilliam, T. A. and M. Sato. 1955. The distribution of myelin on nerve fibres from Pacinian corpuscles. J. Physiol. London 129 : 167-176.

49. Rexed, B. 1944. The postnatal development of the peripheral nervous system in man. Acta Psychiat. Suppl. 33. 
50. ReXed, B. and G. Ström. 1952. Affcrent nervous connexions of the la... al cervical nucleus. Acta Physiol. Scand. 25 : 219-229.

51. Rudin, D. O. and G. Eisenman. 1954a. The action potential of spinal axons in vitro. J. Gen. Physiol. $37:$ 505-538.

52. Silapovalor, A. I. 1963. Intracellular microelectrode investigation of effect of anesthetics on transmission of excitation in the spinal cord. Farmakologiya $i$ Toksikologiya 26: 150 (Federation Proc. Trans. Suppl. $23:$ T 113, 1964).

53. Somjen, G. 1967. Effects of anesthestics on spinal cord of manmals. Aneshesiology $28: 135-143$.

54. Stoney, S. D., W. D. Thompson, and H. Asanuma. 1968. Excitation of pyramiclal tract cells by intracortical microstimulation: The effective extent of the stimulating current. J. Neurophysiol. 31 : 659-669.

55. Swensson, A. 1944. The postnatal development of the peripheral nervous system in man. Acta Psychiat. Suppl. $33: 111$.

56. TASAKI, I. 1964. A new measurement of action currents developed by single nodes of Ranvier. J. Neurophysiol. 27: 1199-1206.

57. TASAKI, I. 1952. Properties of myelinated fibers in frog sciatic nerve and in spinal cord as examined with micro-electrodes. Japan. J, Physiol. $3: 73-94$.

58. TAUb, A. and P. O. Bishop. 1965. The spinocervical tract: Dorsal column linkage, conduction velocity, primary afferent spectrum. Exptl. Neurol. 13:1-21.

59. UdDenberG, N. 1968. Differential localization in dorsal funiculus of fibres originating from different receptors. Exptl. Brain Res. 4: 367-376.

60. Ushiyama, J., K. Korzumi, and C. Mc. Brooks. 1966. Accommodative reactions of neuronal elements in the spinal cord. J. Neutophysiol. 29 : 1028-1045.

61. Vizoso. A. D. and J. Z. Young. 1948. Internode length and fibre diameter in developing and regenerating nerves. J. Anat. $82: 110-134$.

62. Walker, A. E. and T. A. Weaver. 1942. The topical organization and termination of the fibers of the posterior columns in Macaca mulatta. J. Comp. Neurol. 76: $145-158$.

63. WALL, P. D. 1961. Two transmission systems for skin sensation, pp. 475-496. In "Sensory Communication." W. A. Rosenblith [Ed.]. Wiley, New York.

64. Woonbury, J. W. 1952. Direct membrane resting and action potentials from single myelinated nerve fibers. J. Coll. Comp. Physiol. 39: 323-339.

65. Woodbury, J. W. and H. D. Patton. 1952. Electrical activity of single spinal cord elements. Cold Spring Harbor Symp. Quant. Biol. 17 : 185-188.

66. Wyкe, B. 1963. "Brain Function and Metabolic Disorders." Butterworths, London. 